\title{
Retraction Note to: A Method of Balanced Sleep Scheduling in Renewable Wireless Sensor Networks
}

\author{
Maohan Song, Weidang Lu, Hong Peng, Zhijiang Xu, \\ and Jingyu Hua
}

\author{
Retraction Note to: \\ Chapter "A Method of Balanced Sleep Scheduling \\ in Renewable Wireless Sensor Networks" in: \\ L. Meng and Y. Zhang (Eds.): Machine Learning \\ and Intelligent Communications, LNICST 251, \\ https://doi.org/10.1007/978-3-030-00557-3_30
}

The authors have retracted this article [1] because of the following errors which undermine the paper:

1. In Algorithm 1, the output of $S_{\text {awake }}, S_{\text {sleep }}, T H$ and $E_{u}$ are depending on the input of $E_{h, u}, E_{c, u}, E_{r e s, u}, u$ and $\eta$, in which $E_{h, u}$ and $E_{c, u}$ denote the harvesting energy and the energy consumption for the $\mathrm{u}^{\text {th }}$ node, respectively. However, how to calculate and obtain the $\mathrm{E}_{\mathrm{h}, \mathrm{u}}$ and $\mathrm{E}_{\mathrm{c}, \mathrm{u}}$ are not given in the paper.

2. Equation (7) is not correct. $\mathrm{E}_{\mathrm{h}, \mathrm{u}}$ denotes the harvesting energy for the $\mathrm{u}^{\text {th }}$ node, which have already considered $\eta$. $\eta$ should not multiply with $E_{h, u}$ in equation (7). Thus, the result of $E_{u}$ is not correct, which will cause all the following results (the optimal $\tau$, the work mode of the nodes) not correct. It is a very serious problem.

3 . In the simulation results and discussion, the side length of the network is assumed to be $480 \mathrm{~m}$ and the forder length is assumed to be $60 \mathrm{~m}$. This assumption is wrong. It is impossible for the wireless sensor node to harvest energy through wireless energy transfer with such a long distance.

4. The range of $\tau$ shown in the paper is not correct. It should be $(0,1)$. If $\tau$ equals to 1 , which means the node cannot harvest energy.

5. In the simulation results and discussion, the simulation is performed 100 times under the condition of $\tau=0.5$. It is not correct. In the paper, $\tau$ needs to be optimized depending on $\mathrm{TH}$ and $\mathrm{E}_{\mathrm{u}}$. It should not equal to 0.5 . 
In addition to the flaws identified above, the authors would also like to acknowledge the similarities with two publications by different authors [2, 3]which unfortunately were not been referenced in the published manuscript.

[1] Song, M., Lu, W., Peng, H., Xu, Z., Hua, J.: A method of balanced sleep scheduling in renewable wireless sensor networks. In: Meng, L., Zhang, Y. (eds.) MLICOM 2018. LNCS, vol. 251,pp. 293-302. Springer, Cham (2018). https://doi.org/10.1007/978-3030-00557-3_30. Social Informatics and Telecommunications Engineering

[2] Fang, W., Zhang, Z., Mukherjee, M., Shu, L., Zhou, Z.: Energy-utilization aware sleep scheduling in green WSNs for sustainable throughput. In: 43rd Annual Conference of the IEEE Industrial Electronics Society, IECON 2017, pp. 4724-4727. IEEE, October 2017

[3] Fang, W., Mukherjee, M., Shu, L., Zhou, Z., Hancke, G.P.: Energy utilization concerned sleep scheduling in wireless powered communication networks. In: 2017 IEEE International Conference on Communications Workshops (ICC Workshops), pp. 558-563. IEEE, May 2017. 